\title{
Cosmic microwave background anisotropies from plausible double inflation
}

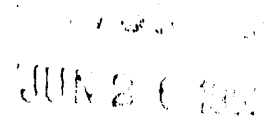

J. N. Fry and Yun Wang

Physics Department, University of Florida, Gainesville, FL 32611

\begin{abstract}
We study the anisotropies in the cosmic microwave background radiation (CMBRR) from certain inflationary models containing two scalar fields, one inflaton and one dilaton. These models can give rise to inflation that occurs in two stages: a first power-law stage during which density fluctuations with a non-scale-invariant power spectrum are generated, followed by a second slow roll-over stage when the dilaton field evolves toward its global ininimum, after the inflaton field has settled down in its true vacuum. The transition can occur on a scale of cosmological interest, $\lambda_{t} \sim 1-1000 \mathrm{Mpc}$. We find that when normalized to yield a quadrupole moment of order $3 \times 10^{-5}$, the features of the power spectrum can siguificantly alcer the angular correlation function of CMBR anisotropies. In particular, a non-scale-invariant spectrum or/and larger amplitudes on large scales can reduce small-scale CMBR anisotropies significantly, while maintaining the observational bound on the quadrupole moment.
\end{abstract}

\section{DISCLAIMER}

This report was prepared as an account of work sponsored by an agency of the United States Government. Neither the United States Government nor any agency thereof, nor any of their employees, makes any warranty, express or implied, or assumes any legal liability or responsibility for the accuracy, completeness, or u.efulness of any information, apparatus, product, or process disclosed, or represents that its use wo!ld not infringe privately owned rights. Reference herein to any specific commercial product, pruness, or service by trade name, trademark, manufacturer, or otherwise does not necessarily constitute or imply its endorsement. recommendation, or favoring by the United States Governenent or any agency thereof. The views and opinions of authors expressed herein do not necessarily state or reflect those of the United States Government or any agency thereof. 
If the seeds of the present structure in the universe were of primordial origin, they should have left an observable imprint on the cosmic microwave background radiation (CMBR). CMBR correlations on scales of more than a few degrees cannot have been affected by causal processes, and thus reflect properties of the universe at the earliest times. Calculations suggest that CMBR anisotropies are just on the threshold of detection [1], if not already in violation of limits. Indeed, an anisotropy of amplitude $1-3 \times 10^{-5}$ has been detected at $1.8 \mathrm{~mm}$ wavelength on scales between $3^{\circ}$ and $22^{\circ}$ but cannot yet be attributed to primordial origin [2]. On the other hand, many theories that have been constructed to implement inflation, which should solve the problems of the standard cosmology (the flatness problem, the horizon problem), also produce seeds for the formation of cosmological structure. Density fluctuations arise due to quantum fluctuations in the relevant scalar field(s) during infiation, and different theories predict a different power spectrum for the density fluctuations. In some inflationary particie physics models, in addition to the standard "inflaton," a second scalar field (which couples to the inflaton) is scmetimes introduced for different purposes. In "double-field inflation" models [3], the second scalar field serves as catalyst to provide an abrupt end to the inflationary epoch. In "double inflation" models [4], the objective is to obtain an interesting power spectrum via the effects of two scaivr fields with differing potentials. In this letter, we use a generic power spectrum with features that appear in certain double inflation models and ask how the angular correlation function of CMBR anisotropies vary with the features. In the rernainder of this paper, first, we discuss the particle physics models that give rise to the desired spectrum. Then we present the main results of the paper, the angular correlation functions from numerical simulation as we vary the features within the reasonable range. Lastly, we conclude and discuss the possible implications of our result. 
Consider theories with a generic form of low energy effective action as follows:

$$
\begin{aligned}
S[g, \phi, \sigma]=\int d^{4} x \sqrt{-g}\left[-\frac{R}{16 \pi G_{N}}\right. & +\frac{1}{2} g^{\mu \nu} \partial_{\mu} \sigma \partial_{\nu} \sigma+F(\sigma) \frac{1}{2} g^{\mu \nu} \partial_{\mu} \phi \partial_{\nu} \phi \\
& -G(\sigma) V(\phi)-f(\phi) V(\sigma)+\Lambda]
\end{aligned}
$$

where $\phi$ is the "inflaton," with potential $V(\phi)$ that allows for a first-order phase transition; $\sigma$ is the "dilaton," the Goldstone boson from some symmetry breaking in the complete theory; $\Lambda$ is some constant. When $\phi$ is trapped in its false vacuum, $\sigma$ varies smoothly; hence we have a period of power-law inflation until the end of the first-order phase transition when $\phi$ reaches its true vacuum. The term with $V(\sigma)$ usually arises due to quantum effects. For instance, it can be generated by the one-loop corrections to the tree-level potential $V(\phi)$. Hence it can be negligible during the inflation caused by the entrapment of $\phi$ in false vacuum. However, after $\phi$ has reached its true vacuum, it is likely that $\sigma$ is still evolving toward its global minimum (provided that the effective potential for $\sigma$ at this stage has a global minimum, which is often the case). If $\sigma$ is sufficiently far (but not too far) away from its minimum, we can have an appropriate amount of additional inflation.

The density fluctuations generated during the first stage of inflation have a nonscale-invariant spectrum, due to the significant dynamical evolution of the dilaton field [5]. The transition scale, denoted as $\lambda_{t}$, corresponds to the time when the first.order phase transition ends, when the bubbles of the true vacuum phase coalesce to form the new phase. Due to the complexities which arise from the bubble collisions, it is still not ciear what happens at this point. However, as soon as the second stage of inflation sets in, the radiation from the reheating at the end of the first stage inflation is rapidly red-shifted away, so we may compute the density fluctuations generated during the second stage of inflation. The amplitudes of the power spectrum for scales $\lambda>\lambda_{t}$ and $\lambda\left\langle\lambda_{t}\right.$ need not match at $\lambda_{t}$, since they have different dependence on the parameters in the theory. 
Fui. [6] proposed a "plausible double inflation," which has all the qualities described above. Although similar power spertra had been discussed previously [4], the model of [6] is more plausible in the sense that the features in the power spectrum (a cosmologically interesting transition scale $\lambda_{t} \sim 1-1000 \mathrm{Mpc}$, a significant step in amplitude at $\lambda_{t}$ ) occur without fining tuning of the parameters in the theory.

To study how such features in the power spectrum affect the angular correlation function of CMBR anisotropy, we consider a generic non-scale-invariant power spectrum with a possible step in amplitude at an interesting transition scale $(1-1000 \mathrm{Mpc})$. At a fixed moment of time in the late (matter-dominated) universe, we have

$$
P(k)=\left\{\begin{aligned}
A_{t} k^{1-2 \delta} T^{2}(k), & \lambda>\lambda_{t}\left(k<k_{t}\right) ; \\
k^{1-2 \epsilon} T^{2}(k), & \lambda<\lambda_{t}\left(k>k_{t}\right) .
\end{aligned}\right.
$$

A scale-invariant spectrum, with fixed amplitude on the scale of the horizon, leads to $P(k) \sim k$ at a fixed mornent of time. The factor $k^{-2 \delta}$ refiects non-scale-invariance on the horizon. In generic extended-inflationary models, $\delta=4 /(2 \omega-1)$, where $\omega$ is a Brans-Dicke-like parameter [5]. In models with non-linearly realized scale invariance [6], $\delta=1 /\left(\pi \hat{f}^{2}-1\right)$, with $\hat{f} \equiv f / M_{P L}$, where $f$ is the decay constant of the dilaton, and $M_{P L}$ is the Planck mass. In the model of Ref. [6], it was shown that for the first stage of inflation to occur, we need $\pi \hat{f}^{2}>1$. To avoid the excess of large bubbles in the absence of the second stage of inflation, we require $\pi \hat{f}^{2} \lesssim 10$; this upper bound is weaker than it seems for our interest. In [6], $\delta=\epsilon$, but $\delta$ and $\epsilon$ could in principle be different. The transfer function $T(k)$ accounts for the changing dynamics of the universe. For modes that enter the horizon at late times when the universe is matter dominated, $T \sim 1$. Modes that enter at early times, when the universe is radiation dominated, grow very little until the time of matter domination, and for large $k, T \sim k^{-2}$. We use the form of $T(k)$ for cold dark matter (CDM) given in Appendix G of Ref. [7]. The ratio of the amplitudes of the power spectrum at the transition scale $\lambda_{k}$ should be calculable in a 
given theory; in [6], it is given by

$$
\frac{P\left(k<k_{t}\right)}{P\left(k>k_{t}\right)} \equiv A_{t}=\frac{128 \pi^{2}\left(\mu / m_{\dot{\phi}}\right)^{4}}{\left|2.19+4 \ln \left(\mu / M_{P L}\right)+S_{B}\right|},
$$

where $\mu$ is the false vacuum scale for the inflaton $\left(\rho_{V} \sim \mu^{4}\right), m_{\tilde{\phi}}$ is the inflaton mass, and $S_{B}$ is the bounce action for the tunneling configuration of the first order phase transition which is responsible for the first stage of inflation. Similarly, the transition scale $\lambda_{t}$ is calculable; in [6], it is

$$
\lambda_{t} \sim \exp \left(N_{S L}-60+\frac{1}{2} S_{B}\right)\left(m_{\bar{\phi}}^{4 / 3} / T_{R H}^{1 / 3} M_{P L}\right) \mathrm{Mpc},
$$

where $N_{S L}$ is the number of $e$-folds from the second stage of inflation, and $T_{R H}$ is the final reheating temperature. Fig. 1 shows $P(k)$ as in (2) for three parameter choices, as indicated in the caption. The solid curve, with $\delta=0$ and $A_{t}=1$, is the standard CDM model. We fix the overall normalization to have the quadrupole moment $Q=3 \times 10^{-5}$ (see below). Although this is an integral over all of $P(k)$, the result of this normalization is very similar to fixing $P(k)$ at $k \sim 4 k_{h}$, where $k_{h}$ corresponds to the horizon distance, $R_{h}=2 c H_{0}^{-1} \approx 12,000 \mathrm{Mpc}$ (we use a Hubble constant $H_{0}=50 \mathrm{~km} \mathrm{~s}^{-1} \mathrm{Mpc}^{-1}$ ).

For scales of interest, the main contribution to CMBR anisotropies arises from fluctuations in the gravitational potential, the so-called Sachs-Wolfe effect [8]. Excluding the unobservable monopole term and the dipole term, dominated by peculiar motion (cf. [1]), CMBR correlations are given by

$$
\begin{aligned}
C(\theta) & =\left\langle\frac{\Delta T}{T}\left(\hat{n}_{1}\right) \frac{\Delta T}{T}\left(\hat{n}_{2}\right)\right\rangle \\
& =\int \frac{d^{3} k}{(2 \pi)^{3}} \frac{P(k)}{c^{2} k^{4}}\left[j_{0}(k r)-j_{0}^{2}\left(k R_{h}\right)-3 \cos \theta j_{1}^{2}\left(k R_{h}\right)\right]
\end{aligned}
$$

where $\cos \theta=\hat{n}_{1} \cdot \hat{n}_{2}, R_{h}$ is the horizon distance, and $r=2 R_{h} \sin (\theta / 2)$.

We evaluate $C(\theta)$ as given by Eq. (5) for the power spectrum given in Eq. (2). The predicted amplitude of $P(k)$ is parameter-dependent. Since COBE has given a limit 
of $Q<3 \times 10^{-5}[9]$, we normalize our results so that the quadrupole moment $Q^{2}$, the coefficient of $P_{2}(\cos \theta)$ in $C(\theta)$, is

$$
Q^{2}=\int \frac{d^{3} k}{(2 \pi)^{3}} \frac{P(k)}{c^{2} k^{4}} 5 j_{2}^{2}\left(k R_{h}\right)=\left(3 \times 10^{-5}\right)^{2} .
$$

This has the effect of fixing $C(\theta)$ at large scales; the variations from one model to another appear mostly at small angles. The results that follow scale with $Q$, and are upper limits on $\Delta T / T$ for a given spectrum.

We show in Figs. 2-4 our results for the root-mean-square temperature tuctuation $\Delta T / T=[2 C(0)-2 C(\theta)]^{1 / 2}$ expected for a single-subtraction experiment, where $\Delta T^{2} \equiv$ $\left\langle\left(T_{1}-T_{2}\right)^{2}\right\rangle$ for a beam-throw (difference angle) $\hat{n}_{1} \cdot \hat{n}_{2}=\cos \theta$, plotted vs. $x=\sin ^{1 / 2}(\theta / 2)$. Fig. 2 shows the effect of varying $\delta$, the deviation from a scale-invariant power spectrum, for $A_{t}=1$. This alone can significantly change $\Delta T / T$. The solid curve is the standard CDM result. Fig. 3 shows the effect of varying $A_{t}$, the amplitude for $\lambda>\lambda_{t}$, for $\delta=0$ and $\lambda_{t}=1000 \mathrm{Mpc}$. The middle (solid) curve is the same as the top curve in Fig. 2. Fig. 4 shows the effect of changing $\lambda_{t}$, for $\delta=0$ and $A_{t}=10$. We have not attempted to filter these results for instrumental resolution, which is crucial for interpreting specific experiments. However, instrumental effects typically do not affect $\Delta T / T$ by as much as the new features of the power spectrum that we consider. The figures clearly indicate that for fixed quadrupole moment, a non-scale-invariant spectrum $(\delta>0)$ or/and larger amplitudes on large scales $\left(A_{t}>1\right)$ can reduce the overall amplitude of small-scale CMBR anisotropies significantly.

In conclusion, we remark that if inflation were indeed realized via inflaton-dilaton interactions, it is likely that the resultant density fluctuations do have such a non-scaleinvariant power spectrum with an intermediate transition scale. Generically, the features in the power spectra desired for large scale structure formation (extra power or/and larger amplitudes on large scales) have the effect of suppressing the CMBR anisotropies. It is 
intriguing to us that in the context of generic "plausible double inflation," the standard CDM scenario need not violate the CMBR anisotropy bounds.

This work was supported in part by the Department of Energy under grant \#DEFG05-86ER40272 at the University of Florida. 


\section{References}

1. N. Vittorio and J. Silk, Astrophys. J. Lett. 285, L39 (1984); J. R. Bond and G. Efstathiou, Astrophys. J. Lett. 285, L45 (1984); K. Górski, Astrophys. J. Lett. 370, L5 (1991); N. Vittorio et al., Astrophys. J. Lett. 372, L1 (1991).

2. S. S. Meyer, E. S. Cheng, and L. A. Page, Astrophys. J. Lett. 371, L7 (1991).

3. A. Linde, Phys. Lett. B 249, 18 (1990); F.C. Adams and K. Freese, Phys. Rev. D 43, 353 (1991).

4. D. S. Salopek, J. R. Bond, and J. M. Bardeen, Phys. Rev. D 40, 1753 (1989); L. A. Kofman and A. D. Linde, Nucl. Phys. B 282, 555 (1987); H. M. Hodges, G. R. Blumenthal, L. A. Kofrnan, and J. R. Primack, Nucl. Phys. B 335197 (1990); J. Silk and M. S. Turner, Phys. Rev. D 35, 419 (1987).

5. E. W. Kolb, D. S. Salopek, and M. S. Turner, Phys. Rev. D 42, 3925 (1990).

6. R. Holman, E. W. Kolb, S. L. Vadas, and Y. Wang, Phys. Rev. D 43, 3833 (1991); Phys. Lett. B 269, 252 (1991).

7. J. Bardeen, J. R. Bond, N. Kaiser, and A. Szalay, Astrophys. J. 304, 15 (1986).

8. R. K. Sachs and A. M. Wolfe, Astrophys. J. 147, 73 (1967).

9. G. F. Smoot et al., Astrophys. J. Lett. 371, L1 (1991). 


\section{Figure Captions}

FIG. 1. The power spectrum $P(k)$ plotted vs. $k$ for the standard CDM model, $\delta=0$ and $A_{t}=1$ (solid line); $P(k)$ with $\delta=1 / 16, A_{t}=3.16$, and $\lambda_{t}=1000 \mathrm{Mpc}$ (long-dashed line); $P(k)$ with $\delta=1 / 8, A_{t}=3.16 \lambda_{t}=100 \mathrm{Mpc}$ (short-dashed line).

FIG. 2. Root mean square $\Delta T / T=[2 C(0)-2 C(\theta)]^{1 / 2}$ for single-subtraction anisotropy measurements plotted as a function of $x=\sin ^{1 / 2}(\theta / 2)$ for models with $A_{t}=1$ and $\delta=0$ (solid line), $\delta=1 / 8$ (short-dash line), $\delta=1 / 4$ (long-dash line), $\delta=3 / 8$ (dot-short-dash line), and $\delta=1 / 2$ (dot-long-dash line). The beam-throw $\theta$ is indicated at the top of the figure.

FIG. 3. Root mean square $\Delta T / T=[2 C(0)-2 C(\theta)]^{1 / 2}$ as a function of $x=\sin ^{1 / 2}(\theta / 2)$ for models with $\delta=0, \lambda_{t}=1000 \mathrm{Mpc}$, and $A_{t}=0.1$ (dot-long-dash line), $A_{t}=0.32$ (dotshort-dash line), $A_{t}=1$ (solid line), $A_{t}=3.2$ (short-dash line), and $A_{t}=10$ (long-dash line).

FIG. 4. Root mean square $\Delta T / T=[2 C(0)-2 C(\theta)]^{1 / 2}$ as a function of $\lambda_{t}$ for models with $A_{t}=10, \delta=0$, plotted for $x=\sin ^{1 / 2}(\theta / 2)=0.2$ (dot-long-dash line), $x=0.4$ (dot-short-dash line), $x=0.6$ (long-dash line), $x=0.8$ (short-dash line), and $x=1.0$ (solid line). 


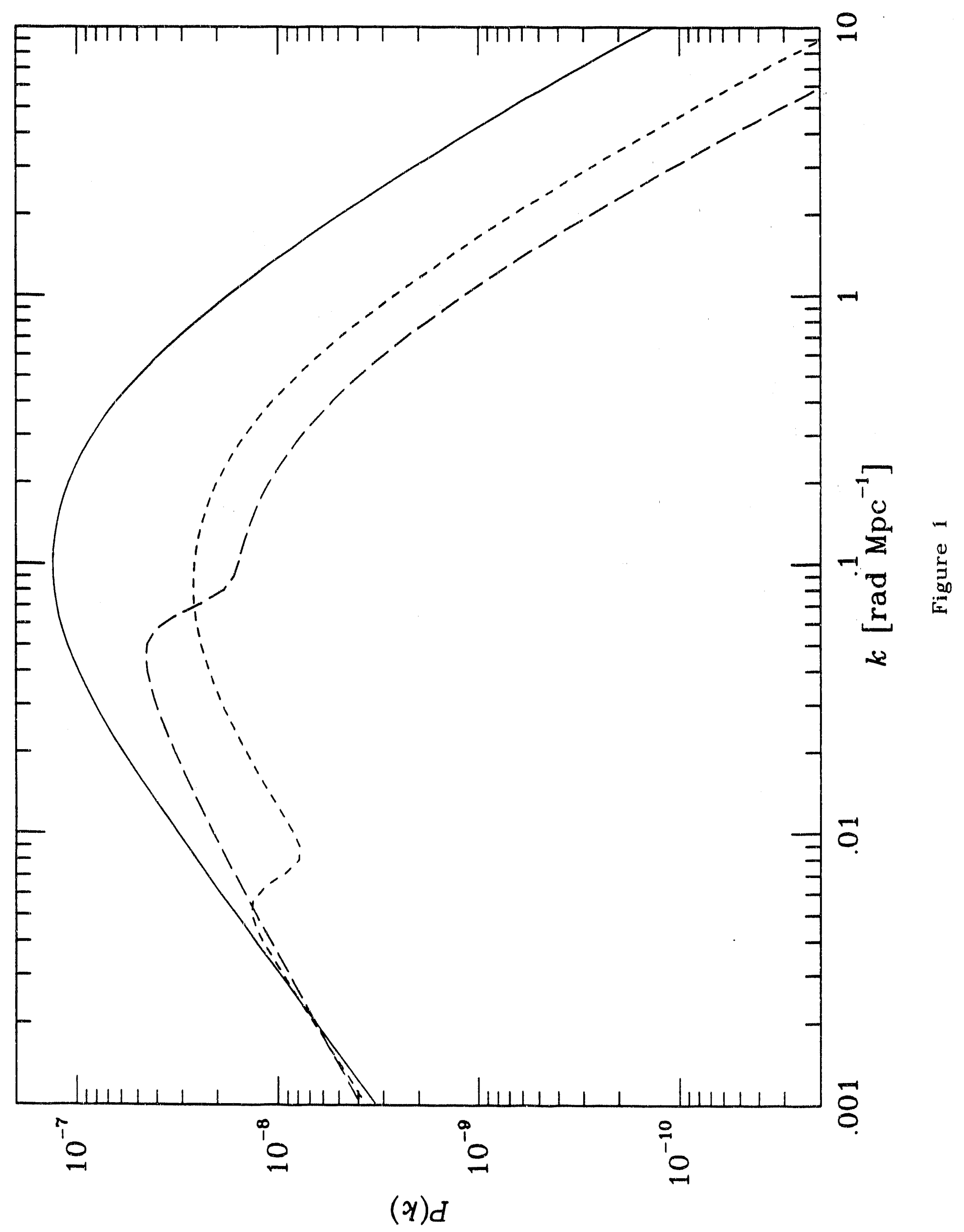




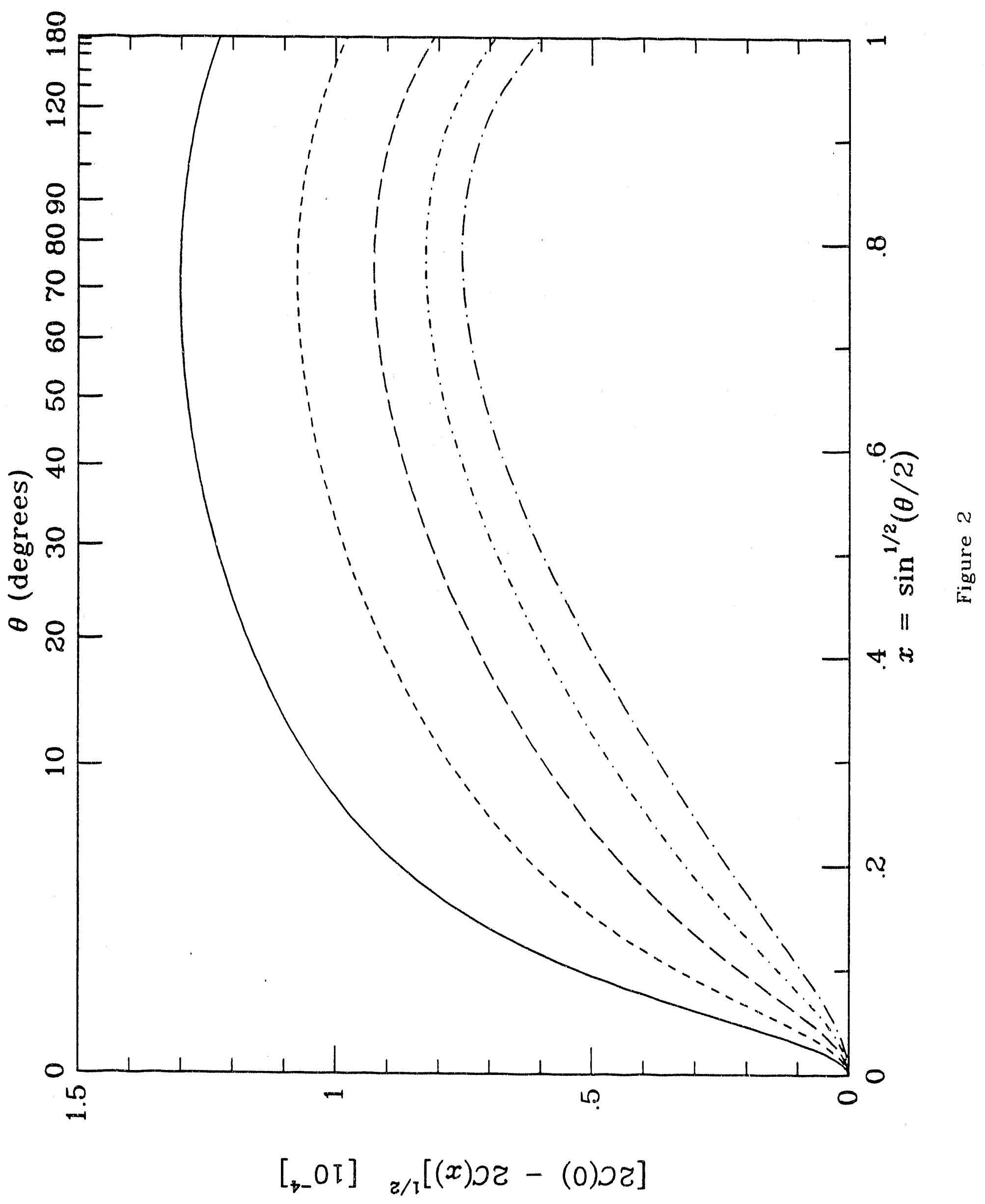




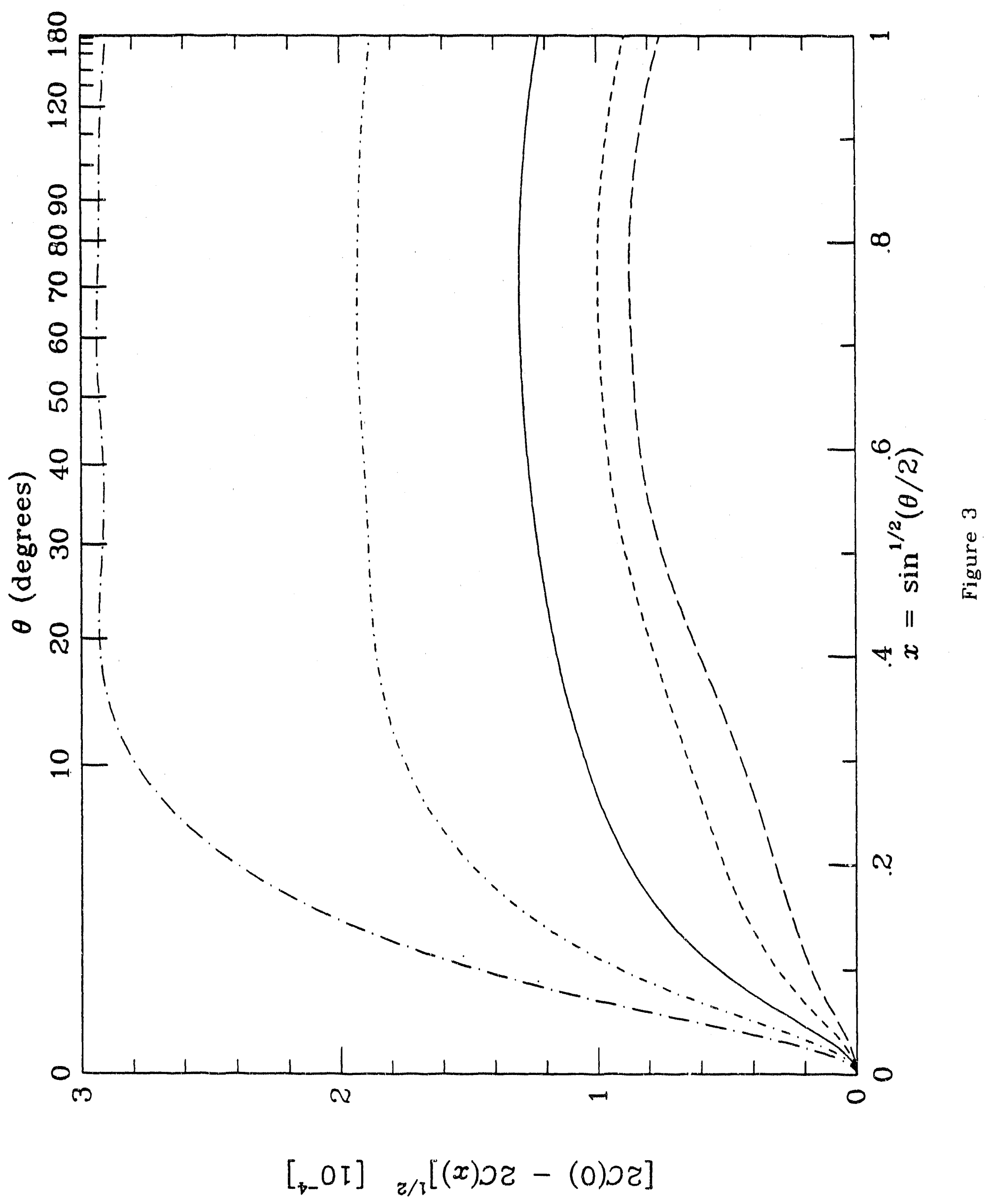




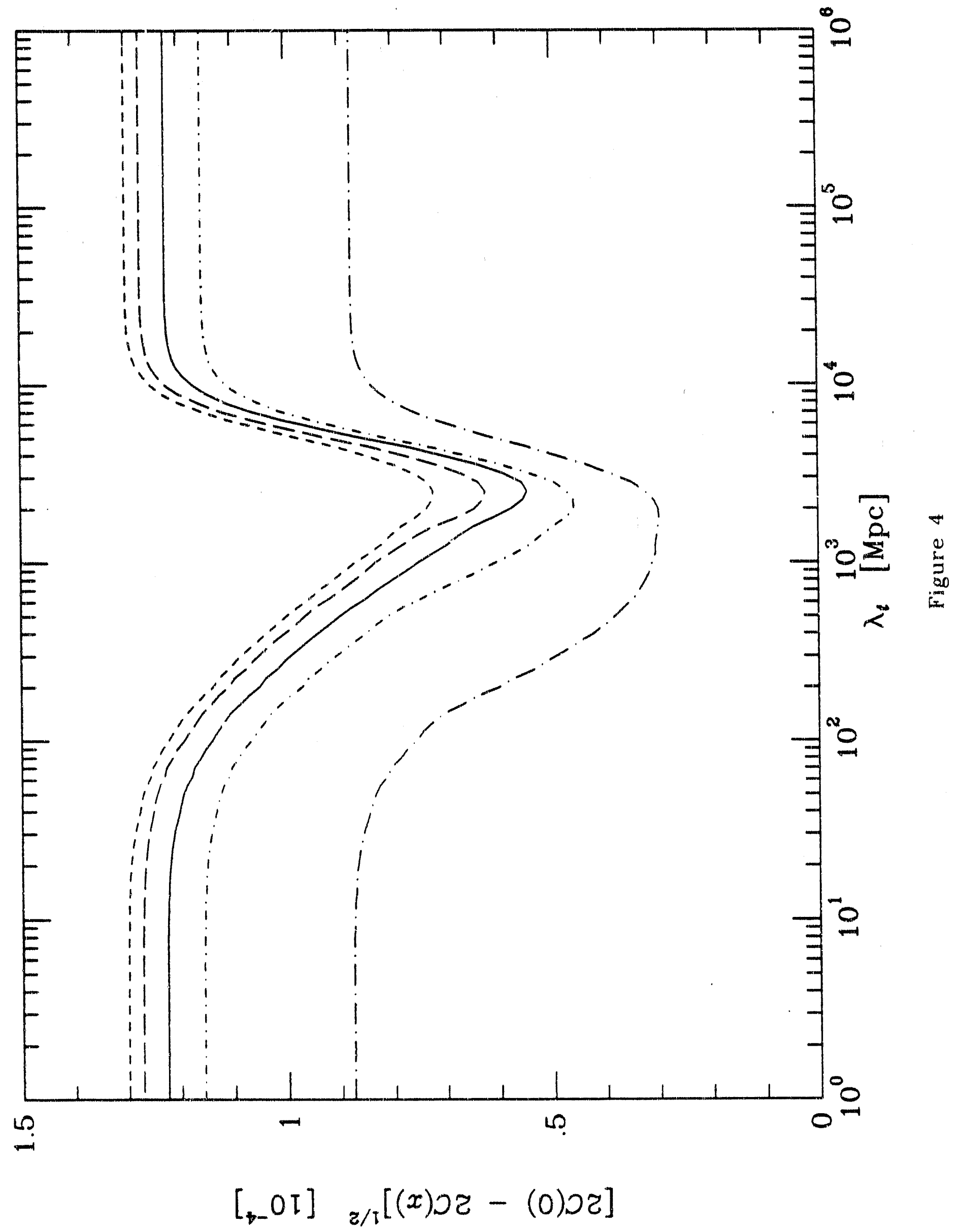



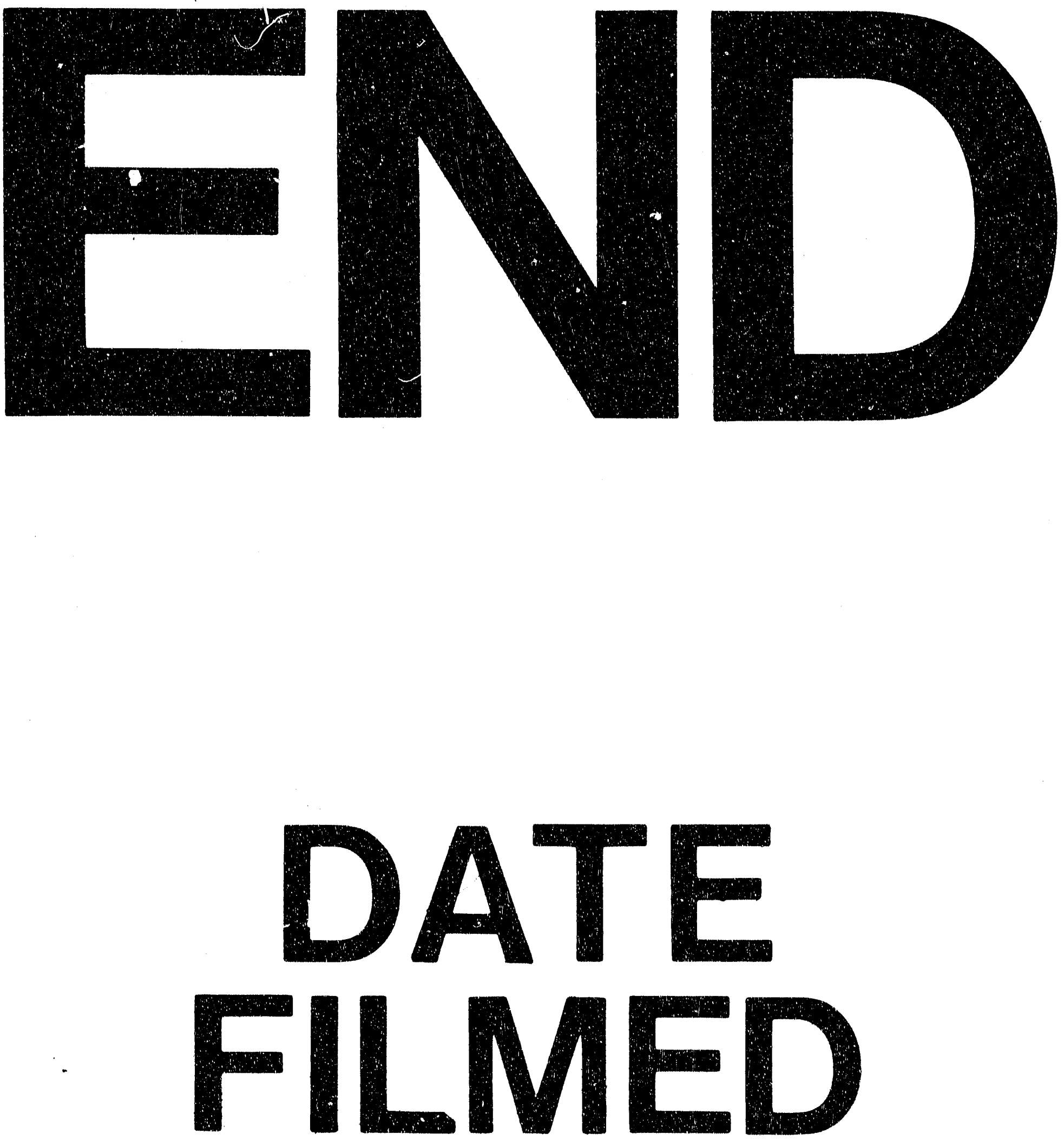

$\$$

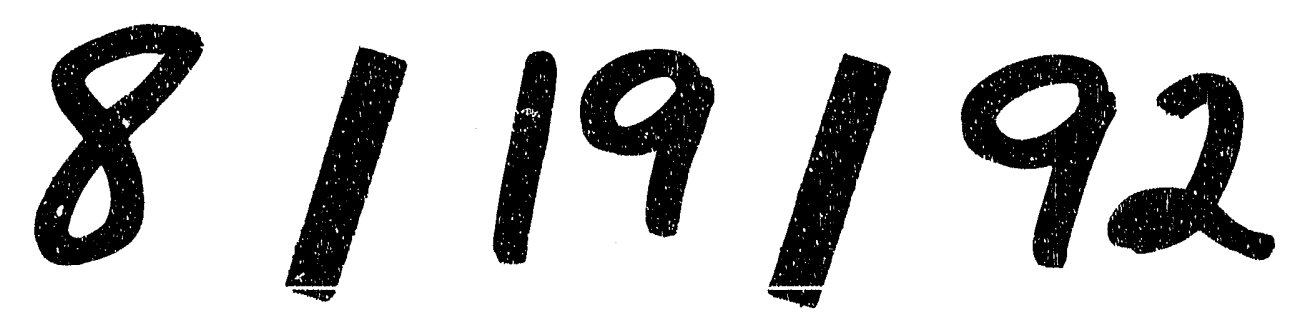


